Doug Geisler, Eva K. Grebel, and Dante Minniti, eds.

\title{
Surface Photometry of Star Clusters in the Dwarf Elliptical Galaxies NGC 185 and NGC 205
}

\author{
Sang Chul Kim, Myung Gyoon Lee \\ Astronomy Program, SEES, Seoul National University
}

Seoul 151-742, Korea

Doug Geisler

Departamento de Física, Grupo de Astronomía, Universidad de Concepción, Casilla 160-C, Concepción, Chile

Ata Sarajedini

Astronomy Department, University of Florida, Gainesville, FL 32611-2055, USA

Taft E. Armandroff

National Optical Astronomy Observatory, P.O. Box 26732, Tucson, Arizona 85726, USA

Gary S. Da Costa

Mount Stromlo and Siding Spring Observatories, The Australian National University, ACT 2611, Australia

\begin{abstract}
We present the surface photometry of star clusters in the nearby dwarf elliptical galaxies NGC 185 and NGC 205, obtained from deep HST WFPC2 F555W $(V)$ and F814W $(I)$ images. We have obtained surface brightness and color profiles of six star clusters in NGC 185, seven star clusters in NGC 205, and one recently discovered non-stellar object in NGC 205. The surface brightness profiles of ten star clusters are fitted well by the King model, and those of four star clusters are fitted well by the power-law. Three out of ten star clusters fitted well with King model show signs of tidal tails.
\end{abstract}

It is essential to understand the structures of star clusters in studying the dynamical evolution of star clusters. There have been several studies on the structures of star clusters in nearby galaxies (LMC, SMC, Fornax dwarf spheroidal, WLM, M31, and NGC 5128). However, there is to date no study of the structures of star clusters in dwarf elliptical galaxies.

We present, for the first time, the surface photometry results of star clusters in the nearby dwarf elliptical galaxies NGC 185 and NGC 205, obtained from deep HST WFPC2 F555W and F814W images. Surface photometry was obtained using concentric annular apertures. Each concentric annular aperture was divided into eight sectors to derive the mean surface brightness and error. 


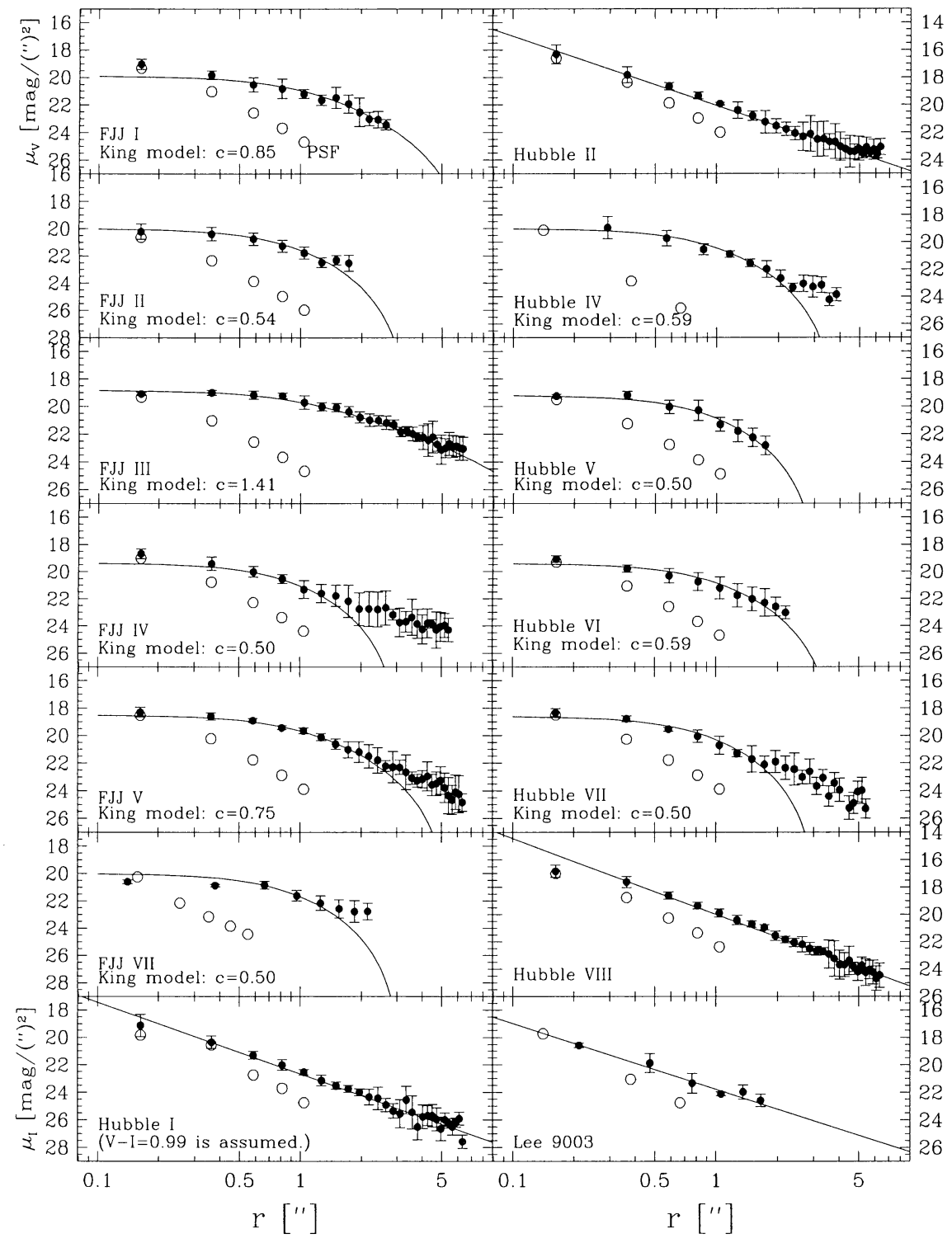

Figure 1. F555W $(V)$ surface brightness profiles of the star clusters in NGC 185 and NGC 205 are shown with solid circles. F814W $(I)$ surface brightness profiles are shown for Hubble I. Open circles represent point spread function (PSF) profiles. Solid lines represent single-mass isotropic King models or a power-law fit to the data. The central concentration parameter $c$ is indicated for King model clusters. 


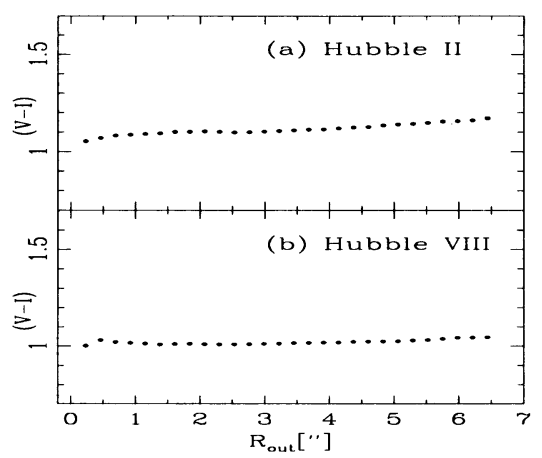

Figure 2. Integrated color profiles of two selected star clusters (a) Hubble II and (b) Hubble VIII in NGC 205.

Surface brightness profiles of six star clusters in NGC 185 (FJJ I, FJJ II, FJJ III, FJJ IV, FJJ V and FJJ VII) and eight star clusters in NGC 205 (Hubble I, Hubble II, Hubble IV, Hubble V, Hubble VI, Hubble VII, Hubble VIII and Lee 9003) are shown in Figure 1. Hubble I has only F814W band images due to spacecraft problems. During his study of stellar populations in the central region of NGC 205, Lee (1996) has noticed that Lee 9003 is a non-stellar object in the CFHT CCD images. Lee 9003 was covered in the WF4 chip of our Hubble $\mathrm{V}$ field, and is found to be a star cluster candidate.

From Figure 1, it is found that seven star clusters (FJJ I, FJJ II, FJJ III, FJJ VII, Hubble IV, Hubble V, and Hubble VI) have surface brightness profiles well fitted by King (1966) model, three star clusters (FJJ IV, FJJ V, and Hubble VII) have those with King model and tidal tails, and four star clusters (Hubble I, Hubble II, Hubble VIII, and Lee 9003) have those well fitted by power-law profiles.

Integrated color profiles are obtained for all the star clusters. All the star clusters show little, if any, radial color gradient. Figure 2 shows the color profiles of two selected star clusters (Hubble II and Hubble VIII).

Integrated photometry shows that the total magnitude $\left(M_{V}\right)$ of the clusters range from -5.6 to $-8.3 \mathrm{mag}$, adopting the distance modulus $(m-M)_{0}=23.96$ for NGC 185 and $(m-M)_{0}=24.59$ for NGC 205. Mean $M_{V}$ values for star clusters is derived to be $\overline{M_{V}}=-6.79 \pm 1.01 \mathrm{mag}$ for NGC 185 , and $\overline{M_{V}}=$ $-7.08 \pm 0.93 \mathrm{mag}$ for NGC $205(-7.29 \pm 0.80 \mathrm{mag}$ without Lee 9003$)$. This shows that the mean magnitudes of the star clusters in NGC 185 and NGC 205 are somewhat fainter than those of our Galaxy.

Acknowledgments. This research is supported in part by the MOST/ KISTEP International Collaboration Research Program (1-99-009).

\section{References}

King, I. R. 1966, AJ, 71, 64

Lee, M. G. 1996, AJ, 112, 1438 